\title{
Dislocation Density-Dependent Quality Factors in InGaN Quantum Dot Containing Microdisks
}

\section{Citation}

El-Ella, H. A. R., F. Rol, M. J. Kappers, K. J. Russell, E. L. Hu, and R. A. Oliver. 2011. Dislocation density-dependent quality factors in InGaN quantum dot containing microdisks. Applied Physics Letters 98 (13): 131909.

\section{Published Version}

doi:10.1063/1.3567545

\section{Permanent link}

http://nrs.harvard.edu/urn-3:HUL.InstRepos:11006842

\section{Terms of Use}

This article was downloaded from Harvard University's DASH repository, and is made available under the terms and conditions applicable to Open Access Policy Articles, as set forth at http:// nrs.harvard.edu/urn-3:HUL.InstRepos:dash.current.terms-of-use\#OAP

\section{Share Your Story}

The Harvard community has made this article openly available.

Please share how this access benefits you. Submit a story.

\section{Accessibility}




\title{
Dislocation density-dependent quality factors in InGaN quantum dot containing microdisks
}

\author{
H.A.R. El-Ellaa), M.J. Kappers, R.A.Oliver \\ Department of Materials Science and Metallurgy, University of Cambridge, Pembroke Street, Cambridge CB2 $3 Q Z$, UK \\ F. Rol, K. Russell, E.L. Hu \\ School of Engineering and Applied Sciences, Harvard University, Oxford Street, Cambridge MA 02138, USA
}

Microdisks incorporating InGaN quantum dots were fabricated using $\mathrm{SiO}_{2}$ microspheres as a hard mask in conjunction with a photoelectrochemical etch step from a structure containing a sacrificial InGaN/InGaN super-lattice. Formation of microdisks from two near-identical structures with differing dislocation densities was carried out and investigated using micro-photoluminescence. This confirmed the existence of quantum dots through the presence of resolution limited spectral lines and showed a clear correlation between the resulting modes quality factors and the dislocation densities within the disks. The disks with higher dislocation densities showed up to $80 \%$ lower quality factors than the low dislocation density disks.

There has been much recent progress in the demonstration of cavity quantum electrodynamics (CQED) in the solid state using modal engineering of high quality optical cavities to dramatically alter the optical behaviour of emitters embedded within. Weak coupling of emitter and cavity can produce controlled single photon emission and ultra-low threshold optical sources $^{1,2,3}$, while in the limit of strong emitter-cavity coupling new quantum mechanical states have been observed ${ }^{4,5,6,7}$ that could serve as the basis for quantum information processing ${ }^{8}$. Although CQED has been explored in a variety of semiconductor materials, there has been relatively little application to the III-nitrides, although their high-band gaps, large exciton binding energies and possible room temperature implementation of CQED effects provide strong motivation to do $\mathrm{SO}^{9}$.

InGaN quantum wells (QW) have been incorporated as the active layers within microdisks ${ }^{10}$ and photonic crystals ${ }^{11}$; generally the $Q$ 's $(Q=\lambda / \delta \lambda)$ of these microcavities have been modest $(\sim 4000)$, although low threshold lasing has been observed in microdisks ${ }^{12}$. The incorporation of quantum dot (QD) active layers promises lower re-absorption (hence loss) than for QW structures, and thus the possibility of achieving higher quality emitter-cavity coupling ${ }^{13}$. InGaN QD active layers have thus far only been reported within pillar cavities with a reported $Q$ not exceeding $280{ }^{14}$. Efforts to explore other geometries, such as microdisks and photonic crystals, encounter the challenges of obtaining high quality InGaN QDs in heterostructures that have also been designed to allow sufficient optical isolation of the cavities. This paper reports the successful growth and fabrication of InGaN QD-containing microdisks and highlights a correlation between the material quality (dislocation density) and the obtained average $Q$ values.

Microdisks were fabricated through a band gap selective photoelectrochemical etch (PECE) in conjunction with a sacrificial super-lattice (SSL), from structures grown on 2 in. c-plane $\mathrm{GaN} / \mathrm{Al}_{2} \mathrm{O}_{3}$ pseudo-substrates by metalorganic vapour phase epitaxy (the pseudo-substrate growth method is described in Ref. 15). The structure design is intended for the creation of undercut microdisks, and consists of an $\operatorname{In}_{\mathrm{x}} \mathrm{Ga}_{1-\mathrm{x}} \mathrm{N} / \operatorname{In}_{\mathrm{y}} \mathrm{Ga}_{1-\mathrm{y}} \mathrm{N}$ SSL grown on an n-doped GaN pseudo-substrate, capped by a thin $(\sim 10 \mathrm{~nm})$ layer of $\mathrm{GaN}$ followed by an $\mathrm{Al}_{0.19} \mathrm{Ga}_{0.81} \mathrm{~N}$ etch stop layer and a $\mathrm{GaN}$ membrane containing an InGaN

\footnotetext{
a) Electronic mail: he234@,cam.ac.uk
} 
QD layer. As there is a strong inherent polarization in $c$-plane nitrides, the super-lattice is intended to modulate the bandedge and ensure a homogenous distribution of photogenerated holes across the SSL surface during the PECE process. The $\mathrm{In}_{0.2} \mathrm{Ga}_{0.8} \mathrm{~N}$ QD layer was grown through a self-assembled droplet epitaxy method which is described in more detail in Ref. 16. The In content of this QD layer is nominally 20\%, but usually varies throughout the layer. The QD-containing microdisk consists of all the layers above the SSL, which serves as the post. A GaN layer was grown between the AlGaN and SSL in order to protect the InGaN SSL layers from the temperature ramp required prior to the AlGaN growth. Two structures containing different SSL In contents were used for fabrication with $\mathrm{x} / \mathrm{y} \sim 7 \% / 11 \%$ (structure $\mathbf{A}$ ) and $\sim 5 \% / 7 \%$ (structure B). These two structures were grown in order to explore the extent of strain-relaxation and resulting material quality throughout the structure and on the fabricated microdisks. The dislocation density (DD) of both sample $\mathbf{A}$ and $\mathbf{B}$ (table I) was determined through calculating the surface pit density (figure 1) using atomic force microscopy (AFM), in combination with cross sectional transmission electron microscopy. These techniques highlighted the generation of additional dislocations within structure $\mathbf{A}$ due to strain-relaxation resulting from an over-abundance of In in the SSL. A student t-test verified the statistical significance $(p<0.002)$ of a larger DD in $\mathbf{A}\left(\sim 3 \times 10^{9} \mathrm{~cm}^{-2}\right)$ to that in $\mathbf{B}\left(\sim 7 \times 10^{8} \mathrm{~cm}^{-2}\right)$.

Fabrication of microdisks with a diameter of $\sim 1.1 \mu \mathrm{m}$ was carried out by first depositing a protective $5 \mathrm{~nm} \mathrm{SiO}_{2}$ layer on the full structure and dispersing $1 \mu \mathrm{m}$ diameter $\mathrm{SiO}_{2}$ spheres onto its surface. The spheres formed masks for an inductivelycoupled plasma etch in $\mathrm{Cl}_{2} / \mathrm{N}_{2}$ that transferred the pattern $\sim 1.5 \mu \mathrm{m}$ into the full structure, stopping in the GaN pseudosubstrate. Subsequent immersion in 50\% HF removed the masking layer. Contact lithography was then employed to deposit a Ti/Pt cathode grid onto the surface. The resulting mesas were then partially undercut by selectively etching the SSL in $2.4 \mathrm{mM} \mathrm{HCl}$ using PECE (details of the full PECE procedure can be found in Ref. 10).

Micro-photoluminescence $(\mu \mathrm{PL})$ of the microdisks was carried out between room temperature $(\mathrm{RT})$ and $5 \mathrm{~K}$ using a pulsed frequency-doubled Ti-sapphire laser emitting at $360 \mathrm{~nm}$. The laser was passed through a variable-neutral density filter and focused by a microscope objective (numerical aperture $=0.5$ ) to a spot diameter of $\sim 1 \mu \mathrm{m}$. The objective was mounted on a xyz piezoelectric stage with a spatial positioning accuracy of $\sim 5 \mathrm{~nm}$. The vertical emission of microdisks was then collected by the same objective, dispersed through to a $0.5 \mathrm{~m}$ focal length spectrometer with a $1200 \mathrm{lmm}^{-1}$ diffraction grating and collected by a liquid $\mathrm{N}_{2}$ cooled charge coupled device. The microdisks were placed on a cold-finger helium flow cryostat that was mounted on an electronically controlled $x y$ linear translational stage with a spatial positioning accuracy of $\sim 50 \mathrm{~nm}$.

Figure 2 shows $\mu \mathrm{PL}$ spectra from a disk fabricated from material $\mathbf{B}$ at $150 \mathrm{~K}$ and at $5 \mathrm{~K}$, highlighting modes with a spacing of $\Delta \lambda \sim 24 \mathrm{~nm}$ and the presence of resolution limited peaks originating from the InGaN QDs. The measured mode spacing correlates well with the theoretical mode spacing of the lowest radial order whispering gallery mode $\left(\Delta \lambda_{n=1}=\lambda_{0}^{2} / 2 r \pi n_{\text {eff }}\right.$, with $n_{\text {eff }}=2.5$ and $\left.r=550 \mathrm{~nm}\right)$ indicating the absence of higher radial order modes and the excellent circularity resulting from the fabrication process. Scanning electron microscopy (SEM) images of sample microdisks created from both $\mathbf{A}$ and $\mathbf{B}$ are shown in figure 3 along with a sample $\mu$ PL scan from each taken at RT. Microdisks fabricated from material $\mathbf{A}$ were observed to exhibit a larger density of whiskers protruding from the disk periphery and underside (in some cases extending as far as the base), reflecting the larger DD. Serving as traps for the photogenerated carriers, and thus locally reducing the etch rate, these dislocations become 'decorated' during the etch, forming whiskers $^{17}$. Direct observation in the SEM of microdisks with $\geq 300 \mathrm{~nm}$ undercuts showed that 9 out of 10 disks created from structure A exhibited whiskers, compared to only 2 out of 10 from structure $\mathbf{B}$.

Measured $Q$ values of microdisks from both samples (figure 4) revealed a larger average value obtained from those of structure $\mathbf{B}$. The difference in mean $Q$ values between $\mathbf{A}$ and $\mathbf{B}$ was verified to be statistically significant through a student $t$ test $\left(p<10^{-4}\right)$ with $\mathbf{A}$ and $\mathbf{B}$ exhibiting a mean $\mathcal{Q}$ of $\sim 2000$ and $\sim 600$ respectively (with a standard deviation of $\sim 450$ and 
$\sim 145$ respectively, see table 1 ). While there are a multitude of possible reasons and mechanisms that can limit measured $Q^{\prime}$, the measured differences in the controlled comparison of microdisks from $\mathbf{A}$ and $\mathbf{B}$ are well accounted for as there is a clear statistical correlation between the DD, the number of observed disks displaying whiskers, and the measured $Q$ values. This strongly suggests that the difference in the $Q$ values is a result of the material DD (and therefore material quality).

However, the mechanism by which these dislocations degrade the $Q$ values is not clear and needs to be investigated further. In particular, it is difficult to pinpoint due to the possibility of several competing scattering and absorption lossmechanisms attributed to the dislocations themselves. Possible loss mechanisms include scattering from the whiskers, although it is not obvious if this is due to the protruding whiskers themselves or through the possible large scale surface roughness their presence causes. The large surface-volume ratio would also possibly enhance the sensitivity to such surface irregularities as has been often shown in other systems ${ }^{18,19}$. The DD within the microdisk itself may also have a significant contribution to sub-bandgap absorption due to band-bending and expected defect level traps occurring within the bandgap. Less obvious mechanisms such as absorption and scattering within the QD active layer in the presence of an increased DD must also be contemplated and studied, especially considering the fluctuation of In around dislocations as well as the growth method and irregularity of the InGaN QD layer. (As well as a broad QD size and composition distribution, modified droplet epitaxy results in a fragmented underlying QW layer and a large density of irregular features with dimensions similar or larger than that of the confined light).

To summarise, microdisks containing $\operatorname{In}_{0.2} \mathrm{Ga}_{0.8} \mathrm{~N}$ QDs were fabricated via a selective PECE process from two structures with differing dislocation densities. $\mu$ PL of microdisks created from both low and high dislocation density material showed a striking correlation between the $Q$ factors and the dislocation density, with the lower dislocation density material exhibiting higher $Q$ factors. Further study is required, as well as optimization of the cavity structure, in order to measure the degree of scattering- and absorption-based mechanisms attributed to the dislocations themselves (both the protruding whiskers and within the microdisk), and assess their contribution to the overall cavity loss. We believe that this study provides important insights into the influence of the material quality on cavity performance in these materials. These insights are critical in enabling the further integrated optimization of material and cavity design to achieve strong QD-cavity coupling.

This work was supported in part by the NSF Materials World Network (award no. 1008480), the Engineering and Physical Sciences Research Council and the Royal Academy of Engineering. R.A. Oliver would like to acknowledge funding from the Royal Society.

\section{Figure Captions:}

Figure 1: $\quad 1 \mu \mathrm{m} \times 1 \mu \mathrm{m}$ AFM scans of (a) structure $\mathbf{A}$, (b) structure $\mathbf{B}$ showing surface pits associated with threading dislocations.

Figure 2: $\quad \mu \mathrm{PL}$ spectra collected vertically from a $\mu$ disk fabricated from material $\mathbf{B}$ at (a) $150 \mathrm{~K}$ and (b) $5 \mathrm{~K}$, with the modes shaded.

Figure 3: $\quad$ Sample SEM images and RT $\mu$ PL spectra collected from (a,i,ii) material A and (b,i,ii) material $\mathbf{B}$ (inset graphs show Lorentzian fits of the respective modes). Note that (b,i) was tilted at a different angle to (a,i), thereby projecting an image of a thinner disk.

Figure 4: $\quad$ Measured $Q$ values from eight observed modes of $\mu$ disks fabricated from structure $\mathbf{A}(\diamond)$ and structure $\mathbf{B}$ 
Table I:

\begin{tabular}{ccccc}
\hline \hline Structure & In \% in SSL & $\begin{array}{c}\text { Surface Pit } \\
\text { Density }\left(\mathbf{c m}^{-2}\right)\end{array}$ & $\bar{Q}$ & $\sigma(Q)$ \\
\hline A & $6.7 \% / 10.7 \%$ & $\sim(3 \pm 0.4) \times 10^{9}$ & $\sim 600 \pm 50$ & $\sim 145$ \\
B & $5.1 \% / 6.5 \%$ & $\sim(7 \pm 0.7) \times 10^{8}$ & $\sim 2000 \pm 160$ & $\sim 450$ \\
\hline \hline
\end{tabular}

Table I caption: $\quad$ Summary of compositions, pit densities and average $\bar{Q}$ (both with standard error), as well as the standard deviation $(\sigma)$ of the measured $Q$ from eight observed modes shown in figure 4.

\section{References}

P. Michler, A. Kiraz, C. Becher, W.V. Schoenfeld, P.M. Petroff, L. Zhang, E.L. Hu and A. Imamoğlu, Science 290, 2282 (2000)

K. Srinivasan, M. Borselli, T.J. Johnson, P.E. Barclay, O. Painter, A. Stintz and S. Krishna, Appl. Phys. Lett. 86, 151106 (2005)

W.-H. Chang, W.-Y. Chen, H.-S. Chang, T.-P. Hsieh, J.-I. Chyi and T.-M. Hsu, Phys. Rev. Lett. 96, 117401 (2006)

J.P. Reithmaier, G. SęK, A. Löffler, C. Hofmann, S. Kuhn, S Reitzenstein, L.V. Keldysh, V.D. Kulakovskii, T.L. Reinecke and A. Forchel Nature (London) 432, 197 (2004).

T.Yoshie, A. Scherer, J. Hendrickson, G. Khitrova, H.M. Gibbs, G. Rupper, C. Ell, O.B. Shchekin and D.G. Deppe Nature (London) 432 , 200 (2004)

E. Peter, P. Snellart, D. Martou, A. Lemaitre, J. Hours, J.M. Gérard and J. Bloch Phys, Rev. Lett. 95, 067401 (2005)

K. Hennessy, A. Badolato, M. Winger, D. Gerave, M. Atatüre, S. Gulde, S. Fält, E.L. Hu and A. Imamoğlu, Nature (London) 445, 896 (2007)

A. Imamoğlu, D.D. Awschalom, G. Burkard, D.P. DiVincenzo, D. Loss, M. Sherwin and A. Small, Phys. Rev. Lett, 83, 4204 (1999)

G. Christmann, R. Butté, E.Feltin, A. Mouti, P. A. Stadelmann, A. Castiglia, J.-F. Carlin, and N. Grandjean, Phys. Rev. B 77, 085310 (2008)

E.D. Haberer, R. Sharma, C. Meier, A.R. Stonas, S. Nakamura, S.P. DenBaars, E.L. Hu, Appl. Phys. Lett. 855179 (2004)

Y.-S. Choi, C. Meier, K. Hennessy, R. Sharma, E. Haberer, Y. Gao, S. P. DenBaars, S. Nakamura, and E. L. Hu, Appl. Phys. Lett. 87,243101 (2005)

A.C. Tamboli, E.D. Haberer, R. Sharma, K.H. Lee, S. Nakamura and E.L. Hu, Nature Photonics (London) 1, 61 (2007)

B. Gayral, J. M. Gérard, A. Lemaître, C. Dupuis, L. Manin, and J. L. Pelouard Appl. Phys. Lett., 75, 1908 (1999)

K. Sebald, M. Seyfried, J. Kalden, J. Gutowski, H. Dartsch, C. Tessarek, T. Aschenbrenner, S. Figge, C. Kruse, D. Hommel, M. Florian, and F. Jahnke Appl. Phys. Lett. 96, 251906 (2010)

R.A. Oliver, M.J. Kappers, J. Sumner, R. Datta, C.J. Humphreys, J. Cryst. Growth. 289506 (2006)

R.A. Oliver, G.A.D. Briggs, M.J. Kappers. C.J. Humphreys, S. Yasin, J.H. Rice, J.D Smith, R.A. Taylor Appl. Phys. Lett., 83755 (2003)

C. Youtsey, L.T. Romano and I. Adesida, Appl. Phys. Lett. 73, 797 (1998)

R. D. Kekatpure, M. L. Brongersma, Phys Rev A, 78, 023829 (2008)

C.P. Michael, K. Srinivasan, T.J. Johnson, O. Painter, K.H.Lee, K. Hennessy, E.L. Hu, Appl. Phys Lett, 90, 051108 (2007) 


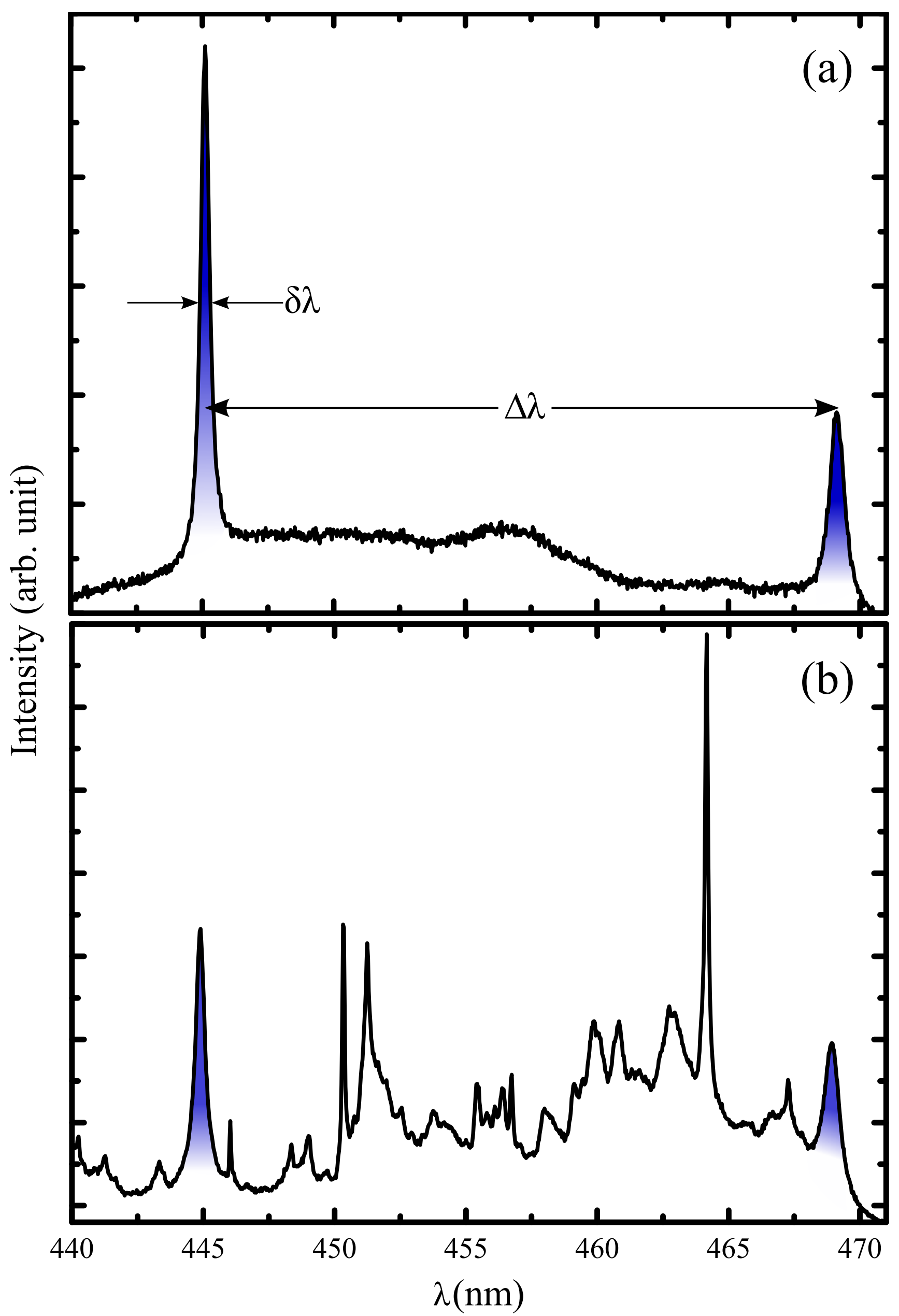




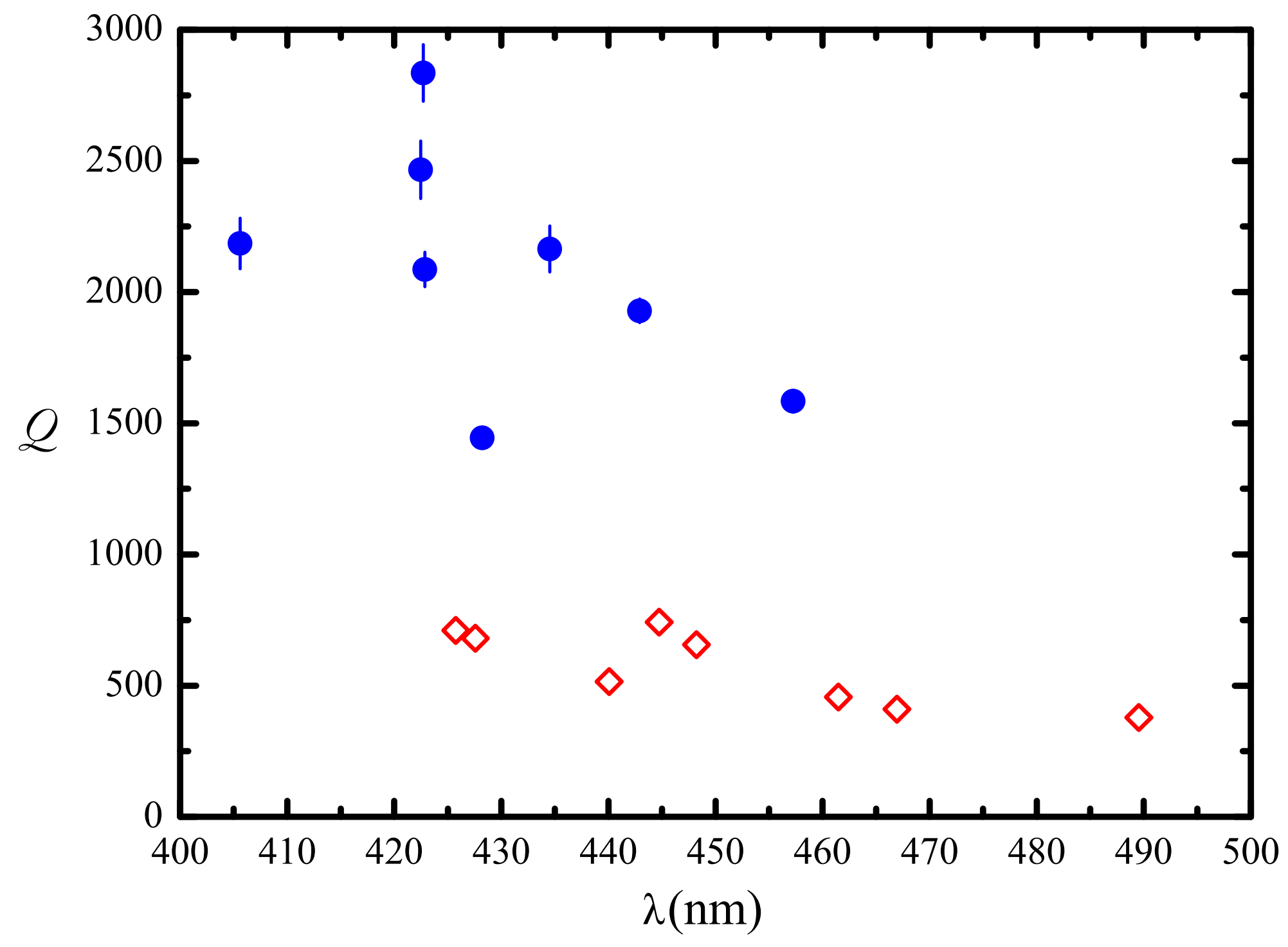

